\title{
Reflections on STEMedicine and path forward to 2021
}

\section{Vincent TORRE}

${ }^{1}$ Neuroscience Area, International School for Advanced Studies (SISSA), 34136 Trieste, Italy.

${ }^{2}$ Institute of Materials (IOM-CNR), Area Science Park, Basovizza, 34149, Trieste, Italy。

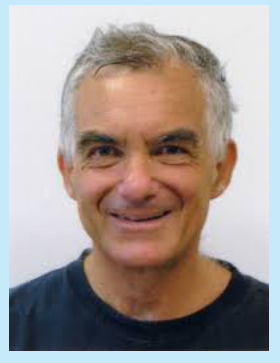

Being a scientist with years of experiences, I've had a vision of a platform for both the scientific and medical communities to share their novel discoveries and technological advances. Now, at the beginning of year 2021, as I look back to my first year as the Editor-in-Chief of STEMedicine, I would like to express my gratitude to all the authors, reviewers and our editorial team for their support and invaluable efforts in bringing this vision on a path to reality.

STEMedicine is an international, peer-reviewed, open access journal and published its inaugural issue with seven articles in January 2020. In its first year, a total of four quarterly issues have been successfully published on time, containing 22 research and review articles, all of which peer reviewed. As I read these articles, I am proud and amazed of their innovative approach and highquality. There are papers from various fields, including cancer, stem cell therapy, neurobiology, inflammatory diseases, etc demonstrating the wide range of science covered by STEMedicine. This breadth of scientific and medical topics enables STEMedicine to connect with researchers from different yet related disciplines.

Besides the broad scope of our articles, STEMedicine also strives to cover the most current research trend. For instance, as the COVID-19 pandemic has just begun to rage throughout the world, two articles on the coronavirus SARS-CoV-2 were published in the 2nd issue of STEMedicine in April $(1,2)$, summarizing the latest understanding of the virus and discussing potential therapy to date. In addition, soon after the news report in Singapore of a British boy's recovery from leukaemia following the experimental chimeric antigen receptor $\mathrm{T}$ (CAR-T) treatment, an article was also published in the third issue covering the progress of CAR-T therapy (3). For this I am greatly thankful for the authors and our editors, especially as the new editorial team is still in the early phase of the learning curve, whose combined efforts have allowed the speedy publication of these timely and excellent articles.

By the end of 2020, STEMedicine has been indexed by Crossref, Directory of Open Access Journals (DOAJ) and Google Scholar. The editorial team and I are confident to make the journal better and bring STEMedicine into the view of an even broader readership in the year of 2021.

Sincerely,

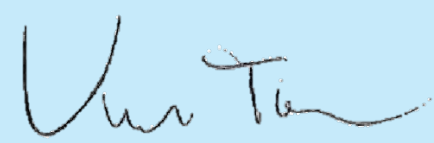

Editor-in-Chief

Vincent Torre

\section{References}

1. Qiu Y, Xu K. Functional studies of the coronavirus nonstructural proteins. STEMedicine. 2020;1(2):e39.

2. Shu C, Huang X, Huang T, Chen L, Yao B, Zhou J, et al. Potential inhibitors for targeting Mpro and Spike of SARS-CoV-2 based on sequence and structural pharmacology analysis. STEMedicine. 2020;1(2):e41.

3. Huang Y, Li L, Liu W, Tang T, Chen L. The progress of CAR-T therapy in cancer and beyond. STEMedicine. 2020;1(3):e47. 